\title{
SAÚDE, DESENVOLVIMENTO E INOVAÇÃO TECNOLÓGICA: NOVA PERSPECTIVA DE ABORDAGEM E DE INVESTIGAÇÃO*
}

\author{
Ana Luiza d'Ávila Viana \\ Fabíola Lana Iozzi \\ Mariana Vercesi de Albuquerque \\ Aylene Bousquat
}

As consequências das reformas de cunho liberal, face à realidade socioeconômica dos países que as adotaram, encorajam um novo debate sobre o papel do Estado no enfrentamento das desigualdades sociais e na condução de políticas de proteção social. Nessa perspectiva, toma força, no Brasil e no mundo, uma nova discussão sobre o conceito de desenvolvimento.

A principal questão posta, no âmbito nacional e internacional, é a de que esse conceito precisa ser resgatado e revisto mediante as experiências acumuladas pelos países, sejam aquelas inspiradas no Estado de bem-estar social, sejam no Estado regulador. Essa revisão é necessária para que se possa formular novas diretrizes sobre o papel do Estado na condução do desenvolvimento e uma nova concepção acerca deste.

A política de saúde deve posicionar-se diante desse desafio teórico e político, pois a saúde é um dos eixos estrutu-

\footnotetext{
* Este artigo é resultante da pesquisa Saúde, desenvolvimento, inovação tecnológica e cooperação regional", financiada pela Fapesp (processo n. 2009/53252-1). Agradecemos ainda ao Prof. Dr. Paulo Elias pelas importantes contribuições ao texto.
} 
rantes das políticas de proteção social e, ao mesmo tempo, um dos setores de maior importância econômica mundial, conformando um verdadeiro complexo econômico-industrial vinculado à saúde.

No campo da investigação de uma concepção renovada sobre saúde e desenvolvimento, devem-se abarcar pelo menos as políticas de ciência, tecnologia e inovação em saúde no Brasil e no estado de São Paulo por seu papel em relação ao complexo industrial desse setor. Nesse procedimento, constituem elementos distintivos a relação entre as políticas de saúde e os padrões de desenvolvimento no Brasil, o estudo de experiências emblemáticas relacionadas com a inovação/incorporação de tecnologias com a finalidade de estabelecer novas possibilidades de articulação entre pesquisa e produção e os fatores que condicionam o desempenho de instituições voltadas para a produção e pesquisa de tecnologias essenciais, componentes do com-

42 plexo econômico industrial da saúde. O enfoque centrado no Estado deve contemplar a inserção das políticas públicas de saúde na proteção social no Brasil considerando-se três grandes fases: 1. Estado desenvolvimentista e protetor (1930 a meados da década de 1980); 2. Estado regulador (1990 a 2003) e 3. novo desenvolvimentismo e novo papel do Estado (2003 aos dias atuais). Nessa abordagem, deve-se privilegiar a investigação da articulação das políticas de desenvolvimento e proteção social.

Essa análise é essencial para que se possa compreender como tal articulação foi possível em outros contextos econômicos e quais lições podem ser aprendidas dessas experiências, de forma a identificar os condicionantes e limites da integração entre as lógicas econômicas e de proteção social, de modo a subsidiar a política de saúde brasileira atual.

A investigação deve contemplar a análise de experiências bem-sucedidas de incorporação de inovações tecnológicas em saúde, relacionadas com diferentes segmentos 
do complexo econômico-industrial da saúde (Ceis) para entender: 1. As diferentes trajetórias de inovação/incorporação de tecnologias no sistema de saúde brasileiro; 2. As articulações que se estabelecem entre os diversos segmentos (público e privado) interessados ou afetados por essas tecnologias e 3. As interdependências econômicas, políticas e tecnológicas que ocorrem no âmbito do sistema de saúde brasileiro, interferindo e condicionando as possibilidades de desenvolvimento desse setor. Assim, neste artigo, o estado de São Paulo ganha destaque no que tange a inovação/ incorporação tecnológica em saúde, pois apresenta a maior concentração de agentes, instituições, investimentos e produtos no âmbito do Ceis no país.

A finalidade desse tipo de abordagem é o de contribuir para a formulação e implementação de políticas públicas capazes de, ao mesmo tempo, induzir o desenvolvimento de setores do Ceis que sejam estratégicos para o Brasil, aumentar o poder de regulação do Estado, fortalecer as instituições públicas que produzem inovações tecnológicas na área da saúde e estabelecer novos padrões de cooperação regional.

No sistema capitalista, a concepção de Estado desenvolvimentista surge nos países periféricos atrelada com a industrialização. De acordo com Mattos (2006, p. 141),

a partir dos anos 1930, o desenvolvimento da economia brasileira esteve voltado para a institucionalização de processos de industrialização. Assim, o desenvolvimento ocorreu centrado, essencialmente, no Estado, ou melhor, com base na forma pela qual o Estado planejou o desenvolvimento econômico através de empresas estatais e, principalmente, por meio da articulação entre capital estatal e capital privado.

Esse tipo de Estado, ao considerar a industrialização como sinônimo de desenvolvimento econômico, abarcava 
uma concepção particular de desenvolvimento social, pois considera este como uma simples consequência daquele processo $^{1}$. Assim, acabava por conceder às políticas sociais um lugar secundário, ou seja, elas ficaram subordinadas aos imperativos da política econômica.

No Brasil, a fundação do sistema nacional de proteção social data de 1930, atrelada ao mercado formal de trabalho (relações e direitos trabalhistas), o que resultou na configuração de um modelo dessa proteção social baseado em uma visão restrita de cidadania, tendo permanecido vigente no país até meados da década de 1980. Assim, a política social nesse período esteve baseada em um modelo corporativo e na extensão de uma "cidadania regulada", como descrito por Santos (1979). Para esse autor, a incorporação dos direitos sociais entre as diversas classes profissionais se dava de maneira fragmentada e diversificada, sendo mais efetiva para as classes mais organizadas e inseridas no processo de 44 modernização econômica.

Segundo Draibe (1997), os sistemas de proteção social presentes nos países latino-americanos durante o século XX eram do tipo meritocrático-particularista, altamente estratificados e corporativos. Segundo a autora, esses sistemas eram "imperfeitos" e "deformados", por conta dos seguintes motivos: 1. estruturais, relacionados com as grandes desigualdades sociais vividas por esses países e pela baixa equidade das políticas sociais e 2. institucionais e organizacionais, referentes ao alto grau de centralização, baixa capacidade regulatória e de implementação de políticas nas esferas subnacionais, corporativismo de grupos profissionais e fraca tradição participativa da sociedade.

Nas discussões sobre políticas sociais não estava contemplada uma concepção abrangente e ampliada acerca

\footnotetext{
${ }^{1} \mathrm{O}$ desenvolvimento social era "entendido como a incorporação dos até então excluídos do mercado formal de trabalho ao processo produtivo, como subproduto do crescimento e do desenvolvimento econômico" (Cohn, 2005, p. 386).
} 
de direitos sociais, implicando restrições e limitações para a expansão de tais políticas. Apesar disso, Machado (2007, p. 56) afirma que

houve certa dinâmica na expansão de algumas políticas sociais, incluindo as de saúde, mesmo no período de autoritarismo militar após 1964. Isso ocorreu sob um modelo distorcido que, na área da saúde, teria propiciado um crescimento do segmento privado prestador de serviços ao Estado, um fortalecimento de mercados de saúde e ampliado a escala das operadoras econômicas na saúde, tanto no âmbito do segmento de assistência médica previdenciária, como no bojo do surgimento de um novo tipo de empresariamento na saúde.

Na década de 1980, a luta pela democratização do regime político fomentou a mobilização de vários segmentos da sociedade também por direitos sociais. Nesse momento, destaca-se o movimento pela reforma sanitária que culminou na implantação do Sistema Único de Saúde (SUS) pela Constituição de 1988, fato estreitamente ligado com a luta pela democratização do país e pela universalidade do acesso à saúde como bem constitutivo de uma concepção de cidadania.

Entretanto, como aponta Machado (2007, p. 57),

se o processo de democratização dos anos 1980 traz a esperança de rupturas mais drásticas voltadas para a construção de uma sociedade mais justa e propicia o desencadeamento de processos de reforma em algumas áreas, a virada liberal-conservadora dos anos 1990 viria a frustrar em grande parte tais expectativas, com repercussões diretas e indiretas sobre a questão e a política social.

As políticas de natureza neoliberal, em vigor no Brasil a partir do início da década de 1990, contribuíram para o 
agravamento do quadro social ao aliar baixo crescimento econômico à manutenção das desigualdades sociais. Nesse contexto de tensionamento do projeto neoliberal, confirmado pelo recente colapso dos mercados financeiros mundiais, insere-se a retomada da discussão acerca de outro modelo de desenvolvimento.

\section{0 resgate do conceito de desenvolvimento}

O esgotamento do neoliberalismo econômico enseja uma revisão sobre a estratégia deste de implementar um único modelo de desenvolvimento, baseado numa receita universal que atribuiu ao Estado o papel de agente meramente regulador da atividade econômica.

Mkandawire (2002) alerta para o surgimento de uma "nova economia do desenvolvimento", baseada em uma nova ideia acerca deste e em experiências acumuladas. Seu objetivo principal seria dar contribuições para aumentar as

46 opções disponíveis à ação do Estado em constestação ao consenso único e à receita universal proposta pelas ideias neoliberais. $\mathrm{O}$ autor destaca que há necessidade, nos tempos atuais, de se criar alternativas ou modelos de desenvolvimento econômico que sejam, ao mesmo tempo, economicamente dinâmicos, politicamente democráticos e socialmente inclusivos.

Por conta da estagnação do crescimento econômico e do aumento das desigualdades sociais nos países que adotaram as políticas neoliberais no período recente de globalização, surgem diversos questionamentos acerca de o desenvolvimento estar vinculado estritamente à economia. Estes vêm sendo propostos pelos novos estudos sobre desenvolvimento que o associam à política econômica e à social (Draibe, 2007a, 2007b), sendo que muitos deles se baseiam também na discussão de conceitos que estão em voga na política mundial, tais como os de democracia, de equidade e de promoção social. 
Uma vertente proposta por Armatya Sen (2000) incorpora a dimensão da democracia e da liberdade ao desenvolvimento. Outra vertente, que ganha força no Brasil e no mundo, é um movimento de resgate da escola estruturalista, a partir de uma releitura de suas teorias com vistas aos conteúdos do período atual. Dentro desta última, há aqueles autores que buscam a construção dessa "nova economia do desenvolvimento" acrescentando as contribuições renovadas da escola institucionalista.

O Brasil entra nessa nova fase desse debate com uma perspectiva muito interessante, pois a escola estruturalista nacional deixou importantes bases teóricas para se pensar um desenvolvimento genuinamente brasileiro, como é o caso da teoria do subdesenvolvimento econômico formulada pelos autores vinculados à Comissão Econômica para a América Latina e Caribe (Cepal $)^{2}$.

A reflexão sobre um projeto nacional de desenvolvimento teve muitos avanços com a escola cepalina. Esta mostrou, sobretudo através das obras de Celso Furtado, como o processo histórico de subdesenvolvimento da formação econômica brasileira e as ideologias e políticas de crescimento e progresso, gradativamente propostas, fomentaram o mito do desenvolvimento.

Celso Furtado (1974) mostra como as raízes da dependência econômica, que caracterizam o subdesenvolvimento, colocam em evidência a ideologia hegemônica do desenvol-

\footnotetext{
${ }^{2}$ A Cepal foi criada em 1948, pelo Conselho Econômico e Social das Nações Unidas (Ecosoc). O economista argentino Raúl Prebish foi um dos grandes líderes dessa instituição e contou com grande colaboração de Celso Furtado para o desenvolvimento de um pensamento voltado para as particularidades do subdesenvolvimento periférico da América Latina (para aprofundamento dessa questão ver Bielschowsky, 2000a, 2000b). Podemos citar, entre outros, alguns autores que têm raízes nas teorias desenvolvidas pela escola cepalina: Maria da Conceição Tavares; Carlos Lessa; José Serra; Luiz Gonzaga de Mello Belluzzo; Luciano Coutinho; João Manuel Cardoso de Melo.
} 
vimento como um mito, e não como uma etapa histórica do progresso de um país ${ }^{3}$.

Atualmente, no Brasil, o que se questiona é a possibilidade da retomada de um projeto nacional de desenvolvimento, ideia que perdeu força no país após a década de 1980 (Coutinho, 2000) ${ }^{4}$. Esse debate tem ocorrido, principalmente, no sentido de resgatar e renovar ideias e proposições estruturalistas da Cepal e relacioná-las às novas teorias sobre inovação, que surgiram no mundo no início da década de 1970.

Luiz Carlos Bresser-Pereira (2007) coloca a necessidade de uma estratégia nacional de desenvolvimento pautada nos seguintes elementos: aumento da capacidade de poupança e investimentos da nação, forma pela qual

3 "Temos assim a prova definitiva de que o desenvolvimento econômico - a ideia de que os povos pobres podem algum dia desfrutar das formas de vida dos atuais povos ricos - é simplesmente irrealizável. Sabemos agora de forma irrefutável que as economias da periferia nunca serão desenvolvidas, no sentido de similares às economias que formam o atual centro do sistema capitalista. Mas como negar que essa ideia tem sido de grande utilidade para mobilizar os povos da periferia e levá-los a aceitar enormes sacrifícios, para legitimar a destruição de formas de cultura arcaicas, para explicar e fazer compreender a necessidade de destruir o meio físico, para justificar formas de dependência que reforçam o caráter predatório do sistema produtivo? Cabe, portanto, afirmar que a ideia de desenvolvimento econômico é um simples mito. Graças a ele tem sido possível desviar as atenções da tarefa básica de identificação das necessidades fundamentais da coletividade e das possibilidades que abre ao homem o avanço da ciência, para concentrá-las em objetos abstratos como são os investimentos, as exportações e o crescimento. A importância principal do modelo The limits to growth é haver contribuído, ainda que não haja sido o seu propósito, para destruir esse mito, seguramente um dos pilares da doutrina que serve de cobertura à dominação dos povos dos países periféricos dentro da nova estrutura do sistema capitalista" (Furtado, 1974, p.75; grifos no original).

4 "Perdeu por causa da crise de financiamento externo que desmontou o padrão de financiamento que dava suporte a um poderoso conjunto de mecanismos de governança macroeconômica e de políticas de Estado. Esses mecanismos permitiam ao Estado brasileiro liderar e articular o processo de desenvolvimento. [...] Subsequente à década de 1980, nos anos 90, o desmonte do projeto de desenvolvimento se concretizou deliberadamente pela adoção, por parte do governo e da elite brasileira, de uma postura liberal, consubstanciada de maneira mais decisiva na segunda metade dos anos 90 , quando a ênfase recaiu exclusivamente na estabilização monetária" (Coutinho, 2000, p. 37). 
incorpora progresso técnico na produção; desenvolvimento do capital humano; aumento da coesão social nacional que resulta em capital social ou em sociedade civil mais forte e democrática e uma política macroeconômica que garanta a saúde financeira do Estado e do Estado-nação, levando a índices de endividamento interno e externo dentro dos limites conservadores.

Seguindo mais para o enfoque do Estado no novo desenvolvimentismo, Siscú, De Paula e Renault (2005) apontam a necessidade de rever os dois modelos anteriores, o de bem-estar social e o neoliberal, para a criação de um novo padrão de desenvolvimento. "A estratégia novo-desenvolvimentista, embora tenha suas origens no 'velho desenvolvimentismo', ainda que com um olhar crítico em alguns aspectos dessa estratégia, busca adequar a estratégia desenvolvimentista aos novos tempos e à realidade brasileira atual" (Siscú, De Paula e Renault, 2004, p. 11). Ou seja, tanto no caso da forte interferência do Estado nas fases mais centralizadas, como na fase neoliberal, a aplicação de cartilhas orientadas pelas instituições internacionais a realidades muito distintas, com resultados bastante insatisfatórios, foi um dos aspectos mais relevantes.

Nesse sentido, a discussão de outro modelo de desenvolvimento tem sido retomada, tanto por intelectuais, como pelos organismos internacionais. Entretanto, é importante atentar para a importação de modelos e para os impactos e condicionamentos que essas ideias, na sua maioria advindas de países com grande força política e econômica, podem resultar nos países periféricos.

Aponta-se a necessidade de avançar na concepção de desenvolvimento que considere não apenas a questão do crescimento econômico e da inovação tecnológica, mas também políticas que embasem um verdadeiro projeto nacional de enfrentamento das desigualdades socioespaciais e de construção de uma cidadania plena. 


\section{Desenvolvimento e saúde}

A partir dos novos questionamentos sobre o conceito de desenvolvimento e sobre o papel do Estado, surge o debate sobre saúde, baseado, sobretudo, na indagação a respeito dos conflitos políticos gerados ao se separar a política econômica (voltada para o Ceis) e a política social (voltada para a proteção social em saúde).

No âmbito internacional, há um movimento de resgate da Declaração de Alma-Ata sobre a necessidade de universalização da saúde, dos Objetivos do Milênio (2000) e do debate sobre o desenvolvimento social. Em 2007, foi realizada a Conferencia Internacional de Salud para el Desarrollo: derechos, hechos y realidades, em Buenos Aires, Argentina, cuja principal preocupação se deu em torno de que a Atenção Primária em Saúde tem que ser fortalecida a partir da compreensão de que o conceito de saúde atual está intimamente relacionado com o desenvolvimento econômico 50 e social.

No âmbito nacional, é importante destacar as reflexões sobre as consequências de se tratar separadamente, no âmbito do SUS, suas características associadas à modernização e à inovação tecnológica e aquelas associadas à universalização do acesso. Atualmente no Brasil, ganham espaço os debates a respeito do complexo econômico industrial da saúde, considerando o processo de inovação tecnológica de base nacional e o Estado no papel de indutor do desenvolvimento de um Ceis brasileiro.

Destaca-se, nessa discussão, o II Seminário Nacional sobre o Complexo Econômico-Industrial da Saúde, realizado pelo BNDES com o intuito de

promover uma ampla discussão e mobilização da sociedade e do setor empresarial com o objetivo de viabilizar fortes mudanças na percepção da saúde como área estratégica de desenvolvimento, introduzindo-se novas políticas, 
novas estratégicas públicas e privadas, novos instrumentos e um novo marco regulatório que permita criar um ambiente político e econômico favorável à inovação e ao desenvolvimento de uma base produtiva local que viabilize o acesso da população aos bens e serviços estratégicos para a saúde (BNDES, 2008).

Neste contexto de significativa mudança na condução da política brasileira de saúde, insere-se a necessidade de nova abordagem que considere a saúde em suas duas dimensões conflitantes: o lócus de acumulação de capital e o de proteção social. Tal enfoque requer o tratamento indissociável das questões relativas à saúde e ao desenvolvimento, levando em consideração as particularidades do território brasileiro.

No entanto, as políticas de modernização relativas a cada projeto de desenvolvimento não atingem de forma igual todos os lugares do território, dependendo das oportunidades que cada lugar oferece para a sua efetivação, a cada momento histórico. Assim, o mito do desenvolvimento se reproduz nas regiões, estados e municípios do país, conforme os interesses públicos e privados de modernização, de acordo com os preceitos de crescimento econômico e de desenvolvimento vigentes.

A política de saúde deve posicionar-se diante do desafio teórico e político do retorno ao debate sobre desenvolvimento e do significado dos mitos acerca deste considerando-se o território brasileiro, por seu papel nas políticas de proteção social e sua dimensão na economia mundial. Ademais, a saúde também se presta para a articulação de projeto de corte nacional, dadas suas relações com as desigualdades socioespaciais e com os anseios de que ela se constitua em direito social.

O SUS explicita claramente as consequências geradas pela separação da política social e da política econômica: 
o sistema se efetiva em todos os lugares do território brasileiro enquanto norma (direito à saúde), mas não enquanto acesso, isto é, não enquanto existência de fato de serviços, equipamentos, profissionais e recursos financeiros (Almeida, 2005). Os lugares que se encontram mais à margem dos processos atuais de modernização e inserção no mercado global são justamente aqueles onde o SUS encontra as maiores dificuldades para a universalização e integralidade das ações (Almeida, 2005; Viana et al., 2007b).

A $13^{\text {a }}$ Conferência Nacional de Saúde, em 2007, constituiu-se em um marco ao debater diretamente a relação saúde e desenvolvimento. A avaliação dos vinte anos de existência do SUS marca a retomada desse debate, partindo de uma visão crítica sobre como a fragmentação - e em determinados aspectos até a dissociação - entre política econômica e política social prejudicou tanto a consolidação do sistema de saúde, como a própria base de produção 52 nacional de equipamentos, insumos e medicamentos para esse sistema.

Em seus discursos de abertura e encerramento da $13^{\mathrm{a}}$ Conferência Nacional de Saúde, o então Ministro da Saúde, José Gomes Temporão, afirma que, apesar das grandes transformações que o Brasil sofreu na sua dinâmica econômica e territorial após a década de 1980 e que resultaram em profundas mudanças na qualidade de vida e na condição de saúde da população, aumentaram as desigualdades entre as regiões do país, quanto à concentração dos serviços e tecnologias de saúde (Brasil, 2007).

Temporão postula que "a saúde tem que ser vista como uma frente de expansão estratégica para a economia e para a política social”. Segundo ele,

a saúde é a única política social que tem uma dualidade fundamental: ela é, ao mesmo tempo, uma política fundamental para a melhoria da qualidade de vida e o desen- 
volvimento da sociedade para melhoria do bem-estar e, de outro lado, ela tem uma dinâmica econômica, um potencial de inovação, de criação de desenvolvimento, riqueza e emprego qualificado que lhe dá uma especificidade. E essa especificidade nunca foi tratada como política de governo e, agora, será (Brasil, 2007).

Um dado importante no discurso do ex-ministro, que se reflete nas políticas lançadas atualmente pelo governo, é a saúde vista com um setor estratégico de inovação tecnológica. Nas palavras de Temporão, "a saúde incorpora todos os setores estratégicos do futuro. Temos no mundo, duas grandes indústrias, que contribuem para o crescimento da pesquisa e da inovação, paradoxalmente, a indústria bélica e a da saúde" (Brasil, 2007). No caso brasileiro, o setor saúde representa os maiores gastos em Ciência e Tecnologia.

Nesse caso particular, segundo Temporão

a saúde não deve ser vista como gasto, ela tem que ser vista como investimento e como fator fundamental do desenvolvimento das sociedades modernas. Ela é a base de um modelo que alia crescimento, inovação, equidade e inclusão social num mesmo processo, numa mesma equação. [...] Desenvolvimento é saúde (Brasil, 2007).

Nesse contexto, ganham importância as políticas federais de ciência, tecnologia e inovação em saúde para o desenvolvimento nacional.

Nota-se atualmente no Brasil uma significativa mudança na política de saúde, com destaque para a discussão, no âmbito do governo federal e sua nova política de desenvolvimento produtivo, sobre a grande potencialidade do Ceis para alavancá-lo no país. Essa nova estratégia destaca-se tanto pelo objetivo de articulação entre vários ministérios na 
elaboração e execução das ações, quanto pela tentativa de integração entre a política econômica e a social.

Segundo o PAC da Saúde:

a saúde possui, assim, duas dimensões que se associam a uma nova aposta para o desenvolvimento do Brasil. É parte da política social e do sistema de proteção social e fonte de geração de riqueza para o País. O direito à saúde articulase com um conjunto altamente dinâmico de atividades econômicas que podem se relacionar virtuosamente num padrão de desenvolvimento que busque o crescimento econômico e a equidade como objetivos complementares (Ministério da Saúde, 2008, p. 3).

Já nas metas do Plano de Desenvolvimento Nacional, destaca-se, como uma das áreas estratégicas, o Ceis. Tal área é ainda citada como estratégica na nova Política Industrial 54 Tecnológica de Comércio Exterior (Pitce) e no PAC de Inovação, conformando uma agenda para o desenvolvimento da base produtiva nacional de bens e serviços em saúde ${ }^{5}$. Desse modo, situa-se como prioridade máxima da política nacional de desenvolvimento, elaborada com base na ampla articulação entre os investimentos públicos e privados (BNDES, 2008).

\footnotetext{
${ }^{5}$ Entre as políticas e seminários realizados nos últimos anos, referentes à inovação e suas implicações para a saúde e ao Ceis como área estratégica, enfatizam-se os seguintes eventos e estudos: PAC Saúde (Programa Mais Saúde: direito de todos), lançado pelo Ministério da Saúde no final de 2007; PAC Inovação (Plano de Ação em Ciência, Tecnologia e Inovação 2007-2010), do Ministério de Ciência e Tecnologia; Política de Desenvolvimento Produtivo (PDP), lançada em maio de 2008 sob coordenação geral do Ministério do Desenvolvimento, Indústria e Comércio Exterior; Política Industrial Tecnológica de Comércio Exterior (Pitce), lançada em março de 2004; Seminário do Complexo Industrial da Saúde, realizado pelo BNDES em maio de 2008; Seminário Inovações para Instituições, organizado pela Secretaria de Assuntos Estratégicos do Governo Federal em agosto de 2008; e Seminário Internacional de Planejamento Territorial no Brasil, iniciativa do Ministério do Planejamento, Orçamento e Gestão em novembro de 2008.
} 
O conceito de Ceis ${ }^{6}$ tem ganhado força por tratar a área da saúde na lógica econômica, evidenciando sua importância para o desenvolvimento nacional.

Gadelha (2001, 2003, 2006) e Gadelha e Costa (2007) chama a atenção para o fato de que o sistema brasileiro de proteção social em saúde não associou suas políticas àquelas diretamente relacionadas ao Ceis. Por conta disso, criou-se uma fragilidade tanto do sistema de proteção social quanto do próprio Ceis, no que se refere ao capital nacional e ao sistema de inovação em saúde.

Viana \& Elias (2007) afirmam que hoje no Brasil convivem de forma complexa e contraditória os processos de formação do Ceis e de mercantilização do acesso e da oferta, por não se constituírem em um mesmo momento histórico. Segundo os autores, essa dissociação impediu o rompimento da natureza dual do sistema de saúde brasileiro, que se caracteriza pela segmentação da clientela e por interesses corporativos no âmbito econômico e tecnológico. Essa dualidade impede a constituição de um sistema que efetive o acesso à saúde como um direito social (Viana et al., 2007a; Viana e Elias, 2007).

A breve análise das principais mudanças na relação entre saúde e desenvolvimento no Brasil esclarece como a dualidade entre interesses econômicos e sociais ainda não

\footnotetext{
${ }^{6}$ Segundo Gadelha (2003, p. 523), o Ceis é "um corte analítico que representa um olhar diferenciado frente à forma tradicional de abordar o setor saúde, representando uma percepção da área como um conjunto interligado de produção de bens e serviços em saúde que se movem no contexto da dinâmica capitalista. De um lado, possui fortes especificidades frente a qualquer outro complexo de atividades; de outro, compartilha a característica geral de se inserir como um conjunto de atividades capitalistas. [...] Com esse olhar, o complexo industrial da saúde pode ser delimitado como um complexo econômico. [...] esse conjunto particular de setores econômicos está inserido num contexto político e institucional bastante particular dado pelas especificidades da área da saúde”. Neste artigo, o autor contextualiza o conceito de Ceis no período atual e cita como trabalhos importantes sobre o tema, como os de Cordeiro (1985) e Negri e Giovanni (2001).
} 
foi superada. Desse modo, a atual tentativa de aproximação desses interesses em uma nova perspectiva de desenvolvimento nacional torna-se positiva, levando-se em consideração que o território brasileiro está impregnado das contradições decorrentes, justamente, dessa longa história de separação dos sentidos do desenvolvimento.

É importante destacar que a partir de 2003, o Ceis se tornou um conceito-chave da política de Ciência, Tecnologia e Inovação do Ministério da Saúde.

A primeira ação nesse sentido foi a criação da Secretaria de Ciência, Tecnologia e Insumos Estratégicos (SCTIE) $^{7}$, em 2003, com a incorporação do Departamento de Ciência e Tecnologia (criado em 2000) e de dois novos departamentos: o Departamento de Economia da Saúde e o Departamento de Assistência Farmacêutica e Insumos Estratégicos.

Logo depois, em 2004, foi aprovado, na 2a Conferên56 cia Nacional de Ciência, Tecnologia e Inovação em Saúde o texto da Política Nacional de Ciência, Tecnologia e Inovação em Saúde (PNCT\&I/S), no qual ficou estabelecido o papel central do Estado na promoção e regulação do Ceis (Brasil, 2006). Esse papel seria exercido por meio das seguintes ações convergentes:

- Apoio à competitividade, financiamento e incentivo à pesquisa e desenvolvimento $(\mathrm{P} \& \mathrm{D})$ nas empresas;

- Uso do poder de compra do Estado em benefício do desenvolvimento do Ceis em negociações internacionais, mantendo situações de flexibilidade no mercado interno para estimular a produção nacional;

- Defesa da propriedade intelectual;

- Incentivo às parcerias (interinstitucionais, interministeriais, entre os entes federados, público-privadas);

\footnotetext{
${ }^{7}$ A proposta de criação da SCTIE surgiu em 1994, durante a 1a Conferência Nacional de Ciência e Tecnologia em Saúde, mas só se efetivou em 2003 (Brasil, 2006).
} 
- Investimento em infraestrutura, sobretudo, para recuperação dos institutos de pesquisa e dos hospitais de ensino.

Além disso, coube ao Ministério da Saúde a função de promover articulações entre instâncias federais voltadas para o desenvolvimento da pesquisa e inovação. A PNCT\&I/S foi elaborada como um componente da Política Nacional de Saúde ${ }^{8}$ e está vinculada com a Política Nacional de Ciência, Tecnologia e Inovação (PNCT\&I), tendo como uma das fontes de recursos financeiros, os fundos setoriais do Ministério da Ciência e Tecnologia.

Dentre as estratégias da PNCT\&I/S estão:

- A sustentação e o fortalecimento do esforço nacional em Ciência, Tecnologia e Inovação em Saúde (CT\&I/S);

- A criação do Sistema Nacional de Inovação em Saúde;

- A construção da Agenda Nacional de Prioridades de Pesquisa em Saúde;

- A superação das desigualdades regionais;

- O aprimoramento da capacidade regulatória do Estado;

- A difusão dos avanços científicos e tecnológicos;

- A formação e capacitação de recursos humanos;

Ainda em 2004, a Pitce incorporou um segmento importante do Ceis: a cadeia produtiva farmacêutica. Vale à pena citar com mais detalhe o Programa de Apoio ao Desenvolvimento da Cadeia Produtiva Farmacêutica (Profarma) do BNDES, lançado em março daquele ano, com a finalidade de apoiar investimentos no país de empresas da cadeia produtiva farmacêutica, incluindo intermediários químicos e extratos vegetais, farmoquímicos e medicamentos para

\footnotetext{
8 "Conjugar o fomento à pesquisa em saúde às necessidades do Sistema Único de Saúde e às demandas da população implica operar transformações tanto no sistema de CTI\&S como no sistema nacional de saúde, legitimando a Política Nacional de Ciência, Tecnologia e Inovação em Saúde como parte integrante da Política Nacional de Saúde" (Conass, 2007, pp. 51-52).
} 
uso humano e outros produtos correlatos voltados para a saúde humana. $\mathrm{O}$ apoio visava às atividades relacionadas à produção de medicamentos (implantação, expansão e/ ou modernização da capacidade produtiva), à Pesquisa, Desenvolvimento e Inovação (infraestrutura de P\&D, inovação incremental e/ou radical de produtos e processos) e ao fortalecimento de empresas nacionais (incorporação, aquisição e/ou fusão de empresas), com o objetivo de: incentivar o aumento da produção de medicamentos para uso humano e seus insumos no país; melhorar os padrões de qualidade desses medicamentos e sua adequação às exigências do órgão regulador nacional; reduzir o déficit comercial da cadeia produtiva; estimular a realização de atividades de pesquisa, desenvolvimento e inovação no país; e fortalecer a posição econômica, financeira, comercial e tecnológica da empresa nacional.

Outra ação do Ministério da Saúde em 2004 foi o lan58 çamento a Agenda Nacional de Prioridades de Pesquisa em Saúde (Brasil, 2004), que serviria de base para a formulação dos editais do Programa de Pesquisa para o SUS (PPSUS) pelas agências estaduais de fomento à pesquisa.

Três anos mais tarde, o governo federal lança o PAC, com o intuito de fortalecer as políticas federais. No mesmo ano, o Ministério da Ciência e Tecnologia lança o Plano de Ação 2007-2010: Ciência, Tecnologia e Inovação para o Desenvolvimento Nacional e o Ministério da Saúde lança o Programa Mais Saúde: Direito de Todos (2008-2011), enfatizando as estratégias voltadas para beneficiar os diversos setores do Ceis.

A partir de 2008, uma série de portarias ministeriais e interministeriais reforça a política de CT\&I/S. Dentre elas, destacam-se:

- A instituição do Programa Nacional para Qualificação, Produção e Inovação em Equipamentos e Materiais de uso em Saúde no Ceis 
- A disposição da lista de produtos estratégicos no âmbito do Sistema Único de Saúde e instituição da Comissão para Revisão e Atualização da referida lista;

- O estabelecimento das diretrizes para a contratação pública de Medicamentos e Fármacos pelo SUS em função da necessidade de incentivar o complexo industrial farmacêutico do país;

- A instituição do Comitê Gestor do Programa Nacional para Qualificação, Produção e Inovação em Equipamentos e Materiais de Uso em Saúde no Ceis, no âmbito da Secretaria de Ciência, Tecnologia e Insumos Estratégicos do Ministério da Saúde;

- A aprovação do regimento interno do Grupo Executivo do Complexo Industrial da Saúde do Ministério da Saúde (Gecis/MS) e a instituição do Fórum Permanente de Articulação com a Sociedade Civil;

- A aprovação do regimento interno da Comissão de Incorporação de Tecnologias do Ministério da Saúde;

- Criação do Departamento do Complexo Industrial e Inovação em Saúde (DCIIS/SE/MS).

Ressalta-se que a criação do Gecis reforça a estratégia do Ministério da Saúde de promover a articulação entre os órgãos federais que conduzem a política de Ciência e Tecnologia (C\&T) do Ministério do Desenvolvimento Indústria e Comercio Exterior e do Ministério de Ciência e Tecnologia. Ao mesmo tempo, o Fórum Permanente de Articulação com a Sociedade Civil, cujo objetivo é auxiliar os debates em relação ao Ceis, reúne os principais membros de todas as associações empresariais com foco na saúde, bem como representantes do SUS.

Até o presente momento, as ações voltadas especificamente para o desenvolvimento do setor de equipamentos e materiais de uso em saúde permaneceram difusas em editais de pesquisa e de fomento à inovação, segundo o tipo de produto (Portaria 978/08, MS) e tipo de inovação (Editais 
Finep e BNDES voltados para as empresas do setor). Não houve a proposição de uma política consolidada para todo o setor, como a exemplo do Profarma.

É importante destacar que o Pacto pela Saúde ${ }^{9}$ inicia um novo momento para a descentralização da política de saúde e para a valorização do papel dos entes estaduais.

Os estados que aderiram ao Pacto pela Saúde tiveram que elaborar seus planos estaduais de saúde em conformidade com as diretrizes desse documento. Nesse processo, surgiu a oportunidade de considerar nos planos as diretrizes contidas na PNCT\&I/S. Destaca-se como um resultado importante dessa incorporação do tema da CT\&I/S, a definição das agendas prioritárias de pesquisa nas fundações estaduais de amparo à pesquisa no Programa de Pesquisa para o SUS ${ }^{10}$ (Editais PPSUS). Todavia, a falta de tradição na gestão de CT\&I/S ainda é um fator que dificulta a atuação do gestor estadual no PPSUS, cujo maior objetivo é dar res60 posta ao sistema local de saúde (Conass, 2007).

A PNCT\&I/S revela uma preocupação do Ministério da Saúde em inserir os entes estaduais e municipais nas ações de promoção do desenvolvimento científico e tecnológico de interesse para o SUS. As Secretarias Estaduais de Saúde, por exemplo, devem criar estruturas e diretrizes para gestão e fomento da CT\&I/S, com apoio do Ministério da Saúde, tais como Conselhos de CT\&I/S, Editais do PPSUS, fortale-

\footnotetext{
${ }_{9}^{9}$ O Pacto pela Saúde (composto pelo Pacto em defesa do SUS, o Pacto pela Vida e o Pacto de Gestão) foi aprovado em 2006 pela Comissão Intergestores Tripartite (CIT), instância de negociação de políticas e ações no âmbito do SUS, contando com representações de gestores das esferas federal (Ministério da Saúde), estadual (Conselho Nacional de Secretários de Saúde - CONASS) e municipal (Conselho Nacional de Secretários Municipais de Saúde - CONASEMS).

${ }^{10}$ É importante destacar que: 1 . "essa ação ampliou uma iniciativa, desencadeada em 2002, de descentralizar recursos aos estados com repasses do Fundo Nacional de Saúde para as Fundações de Amparo à Pesquisa (FAP)" (Conass, 2007, p. 42); 2. "a aproximação entre os sistemas locais de saúde e de ciência e tecnologia, com o trabalho conjunto de FAP e SES possibilitou a produção de conhecimento em consonância com as prioridades e vocações regionais de pesquisa em saúde priorizando a gestão compartilhada de ações" (Conass, 2007, p. 50).
} 
cimento dos hospitais de ensino para o desenvolvimento de $\mathrm{P} \& \mathrm{D}$ em coerência com as necessidades regionais e incentivar as parcerias público-privadas para $\mathrm{P} \& \mathrm{D} / \mathrm{S}$.

Ao mesmo tempo, verifica-se que a PNCT\&I/S diz respeito, acima de tudo, ao papel central do Ministério da Saúde nos processos estratégicos de fortalecimento do Ceis: a regulação da produção e incorporação tecnológica, a criação do sistema nacional de CT\&I/S, estimular o poder de compra do Estado, promover a articulação institucional e intersetorial, definir prioridades para $\mathrm{P} \& \mathrm{D} / \mathrm{S}$, organizar sistema de informações de CT\&I/S, fomentar a formação de redes de CT\&I, incentivar a formação e capacitação de recursos humanos para as atividades de $\mathrm{P} \& D$ e definir as fontes e destinos dos recursos para a PNCT\&I/S.

Com exceção dos avanços obtidos através do PPSUS, citado anteriormente, os mecanismos de desenvolvimento da CT\&I/S ainda permanecem muito verticalizados, isto é, depentendes de decisão da esfera federal, especialmente o que se relaciona ao poder de compra, à regulação e a definição dos incentivos e fontes de recursos para CT\&I/S. Como consequência, os Estados ainda são pouco estimulados a exercerem um papel mais proeminente na formulação de uma visão regional dos processos que envolvem a produção de CT\&I/S.

Os Estados podem apresentar potencial distintivo para estimular as interações entre os agentes do Ceis - os serviços, a indústria e as universidades - sem perder de vista a lógica da política de saúde e a regionalização dos processos de inovação.

Soma-se a isso, o fato de que o processo de inovação e incorporação tecnológica também ocorre a partir da interação de diversos agentes públicos e privados, em cada estado e região. Nesse sentido, considera-se fundamental analisar e discutir o Ceis no contexto dos complexos regionais da saúde. 
O complexo regional da saúde é composto pelas diferentes estruturas, instituições, instâncias e agentes públicos e privados que participam da conformação do sistema e da política de saúde nas regiões de saúde (Cealag, 2007; Viana et al., 2008). Ceis se realiza de diversas maneiras nos lugares e se insere no complexo regional da saúde. Esse conceito de complexo regional incorpora, portanto, os agentes do Ceis (indústria, serviços e universidades) e, também, as operadoras da saúde suplementar, as corporações médicas, os consórcios de saúde e as instâncias de planejamento e gestão pública do sistema de saúde local e regional. A incorporação do Ceis no âmbito do complexo regional possibilita ampliar a análise sobre como as estratégias de inovação em saúde se articulam regionalmente no território e se vinculam à lógica da política desse setor.

Lehoux et al. (2008), analisando os processos de inovação em saúde, chama a atenção para o fato de que há 62 pouca interação entre política de saúde e a pesquisa aplicada a essa área. Atualmente, a política foca a relação entre estes agentes pela análise econômica de evidência de custo/ benefício e risco do uso da tecnologia. Porém, essa análise corresponde ao final do processo da inovação, isto é, às decisões de incorporação tecnológica. Desse modo, o processo de inovação, é pensado pela lógica industrial e não pela lógica da política de saúde. Para autora, é fundamental melhorar as formas de integração desse processo entre indústria e serviços, para que a política de saúde pense a inovação desde o início do processo.

A atuação do Estado como regulador da incorporação tecnológica é insuficiente para estimular a interrelação entre indústria e serviços de saúde para a inovação. De acordo com Lehoux et al. (2008), essa interrelação dependeria da existência de três critérios: 1. a pertinência da inovação em relação às demandas e prioridades em saúde; 2. a aplicabilidade da inovação no sistema de saúde e 3. a 
sustentabilidade da inovação. Os serviços de saúde devem influenciar o processo de inovação desde o início, considerando esses três critérios, e isso contribuiria para que o Estado pudesse avaliar melhor o significado da tecnologia para o sistema de saúde.

No caso brasileiro, o poder de compra do Estado que expressaria um dos aspectos mais estratégicos dessa integração do processo de inovação à política de saúde é atomizado e não atinge suas potencialidades no âmbito da política de CT\&I/S. No entanto, a Portaria 978/08 do Ministério da Saúde constitui-se em ação importante na definição dos produtos estratégicos para a saúde brasileira que devem receber incentivos em CT\&I do poder de compra do Estado. Entretanto, o texto não explora a importância da relação entre indústria, serviços e universidades e não faz menção às estratégias regionais de articulação institucional para o desenvolvimento dessas tecnologias.

A importância de se investigar especialmente o estado de São Paulo resulta da constatação de diversos autores (Fipe e IPT, 2008; Furtado, 2001; ABDI, 2008a, 2008b) de que este apresenta a maior concentração de agentes, instituições, investimentos e produtos no âmbito do Ceis no país. Vale destacar que o Plano Estadual de Saúde de São Paulo (2008-2011), inclui em seus eixos prioritários a política de CT\&I/S.

Em 2008, seguindo as proposições do Eixo IX: Tecnologia e Inovação em Saúde do Plano Estadual de Saúde, a SES-SP instituiu o Conselho Estadual de Ciência, Tecnologia e Inovação em Saúde (CCT\&I/S), através da Resolução n. 26, de 26 de fevereiro de 2008. O CCT\&I/S é composto por representantes dos Institutos estaduais de $\mathrm{P} \& \mathrm{D} / \mathrm{S}$, das Coordenadorias e pelo Secretário Adjunto da SES-SP, por representante das universidades estaduais, da Fundação para o Remédio Popular e das Secretarias Estuduais de Desenvolvimento e de Ensino Superior. A fun- 
ção do Conselho é assessorar o secretário de saúde do estado de São Paulo na formulação e condução da Política de Desenvolvimento Científico, Tecnológico e de Inovação em Saúde no âmbito institucional. Os objetivos do Conselho são: 1. propor a Política de Desenvolvimento Científico, Tecnológico e de Inovação em Saúde; 2. propor programas de ações gerais que operacionalizem e modernizem o sistema de CT\&I da Secretaria de Saúde do estado de São Paulo; 3. definir a Agenda Estadual de pesquisa e desenvolvimento tecnológico em saúde em consonância com a Agenda Nacional de Prioridades em Pesquisa em Saúde; 4. Propor programas estaduais de desenvolvimento científico e tecnológico em saúde em consonância com a Agenda de Prioridades estabelecida; e 5. Identificar fontes de recursos e financiamentos, para pesquisa e desenvolvimento científico, tecnológico e de inovação em Saúde.

Conforme se pode analisar pelas pautas e memórias 64 das reuniões do CCT\&I/S, desde sua criação as reuniões têm ocorrido mensalmente conforme determinado e dentre os assuntos tratados se destacam a formulação e aprovação da agenda estadual de prioridades de pesquisa em saúde, a discussão do Plano Estadual de Saúde em relação à política de CT\&I/S e as necessidades do SUS em São Paulo e a articulação das propostas da Secretaria Estadual de Saúde de São Paulo para CT\&I/S com as diretrizes nacionais e estaduais de CT\&I. Um dos primeiros resultados dos debates no âmbito do CCT\&I/S foi a definição das prioridades de pesquisa em saúde para o edital PPSUS/2009 da Fapesp.

Nesse contexto, o acompanhamento crítico do contexto institucional das políticas de ciência, tecnologia e inovação em saúde no Brasil e no estado de São Paulo se mostra de extrema relevância, principalmente pelo caráter recente das mudanças em curso. 


\section{Inovação tecnológica, regulação e saúde}

Hoje, discussões sobre o conceito de inovação têm sido cada vez mais constantes não só entre intelectuais e empresários, como também nas esferas institucionais e de pesquisa do governo federal. A premissa de que o Brasil precisa dar passos largos em direção a práticas inovadoras vem sendo amplamente discutida, não ficando restrita apenas aos seus aspectos empresariais, que são de suma importância, mas também aos aspectos ligados as instituições e possibilidades de financiamentos que sejam indutores dessa prática.

No momento atual de globalização, práticas inovadoras aparecem como mecanismo sine qua non para manutenção da competitividade externa dos países, bem como para diminuir a ingerência e dependência externa - problemas penosos para países subdesenvolvidos. A área da saúde destaca-se nesse contexto por envolver altos custos e dependência de diversos setores. Além disso, é uma das áreas que concentram maiores investimentos e possibilidades de incremento em inovação, constituindo-se em relevante lócus de acumulação de capital e além de sua importância na dimensão do bem-estar social.

A grande questão que se coloca é como realizar uma nova forma de desenvolvimento que seja fiel aliada da inovação em países que não tenham tradição nessa área, a exemplo do Brasil.

Na área da saúde nos países desenvolvidos, um dos fenômenos mais importantes ocorridos após a Segunda Guerra foi o desenvolvimento de produtos e serviços para os sistemas de saúde, notadamente quanto aos medicamentos, equipamentos médicos e materiais diversos. Diversos aspectos são responsáveis por esse fenômeno, incluindo: 1. a proposição e implementação de políticas científicas e tecnológicas via Estado; 2. a emergência e consolidação da saúde como um direito de cidadania, ampliando o acesso da população aos serviços ofertados; 3. o fortalecimento do 
médico como principal profissional do setor e o desenvolvimento de novos tipos de serviços, diagnósticos, terapias e modalidades assistenciais; 4 . o processo de medicalização da sociedade e 5. a transição demográfica e epidemiológica (aumento da esperança de vida, redução da mortalidade por doenças infecciosas e aumento e diversificação das doenças crônico-degenerativas), implicando aumento da demanda em saúde (Novaes, 2006).

Ao mesmo tempo, observa-se um crescimento da demanda por serviços de alta complexidade tecnológica e a participação cada vez maior do setor privado na oferta desses serviços, especialmente nas grandes metrópoles. A pressão de demanda está relacionada com múltiplos fatores, entre os quais cabe destacar a já citada transição demográfica e epidemiológica, a crescente complexidade tecnológica dos procedimentos diagnósticos e terapêuticos, a pressão exercida pelos grandes conglomerados empresariais do Ceis e o 66 próprio papel dos profissionais médicos, que demandam a realização desses serviços.

Em função do crescimento contínuo dos gastos com saúde, a produção cada vez maior de novas tecnologias e as mudanças no perfil epidemiológico das populações, levando a necessidades diversificadas de atenção, o desenvolvimento de mecanismos de articulação entre os setores envolvidos na produção, incorporação e utilização de tecnologias nos sistemas de saúde tornou-se uma necessidade social e política (Brasil, 2006). De fato, gestores governamentais da área da saúde em diversos países desenvolvidos como os da América do Norte, da Europa Ocidental e a Austrália, a partir do início da década de 1990, passam a considerar a produção e o uso de evidências científicas nas políticas de regulação e nos padrões de incorporação e de utilização de tecnologias.

De fato, a incorporação desordenada e acrítica de inovações tecnológicas em saúde favorece a duplicidade de 
meios para fins idênticos, transferindo custos desnecessários para a sociedade e gerando ineficiência do sistema. Além disso, muitos serviços nessa área, em especial os de média e alta complexidade, precisam ter uma abordagem regional, dado que economia de escala é fundamental para um funcionamento racional dessas unidades e para sua adequada manutenção econômica (Conass, 2007).

As decisões relacionadas à adoção de dada tecnologia são complexas e devem repousar nos mecanismos variados e engenhosos de regulação. As instâncias reguladoras públicas responsáveis pelo registro de tecnologias e sua aprovação para uso comercial devem administrar conflitos de interesses (os econômicos nacionais, os das empresas, os dos gestores e dos profissionais e os da população).

De acordo com Lehoux (2002), o Estado precisa rever (ou reinventar) seu papel na regulação das tecnologias em saúde, para reduzir os efeitos negativos do mercado quanto à oferta de cuidados nessa área, intervir no processo de inovação, de modo a orientar a natureza das tecnologias e seu impacto sobre os custos e considerar os aspectos éticos e sociais engendrados pela utilização do conhecimento técnico-científico moderno, que exige uma tomada de posição coletiva.

Lehoux (2002) coloca a questão de como o Estado pode concretamente orientar e sustentar projetos particulares de $\mathrm{P} \& \mathrm{D}$ no âmbito da saúde e favorecer o uso apropriado das tecnologias. Para essa autora, essa influência pode ser exercida por meio de três diferentes políticas: comerciais, de $\mathrm{P} \& \mathrm{D}$ e de saúde. As políticas comerciais podem influir no financiamento e criação de empresas, principalmente no setor de equipamentos médicos (via créditos e subvenções), as políticas de $\mathrm{P} \& \mathrm{D}$ em geral podem promover o desenvolvimento de tecnologias particulares - que, a curto e médio prazo, levam a transformar os serviços (biotecnologia, telecomunicações, microeletrônica) e as políti- 
cas de saúde -, exercer um impacto direto sobre a oferta de cuidados em saúde, notadamente quanto à regulação da entrada de equipamentos e medicamentos nos sistemas de saúde. Ainda segundo a autora, o desafio, consiste em encontrar um equilíbrio naquilo que parece ser a dimensão esquizofrênica da política, qual seja, tratar de buscar formas para incentivar a consolidação de uma indústria lucrativa e que joga papel importante no desenvolvimento econômico e, ao mesmo tempo, adotar medidas vigorosas de controle das despesas em saúde.

No caso do Brasil, é importante ressaltar a existência de forte dependência tecnológica externa no que diz respeito ao acesso a novos fármacos e medicamentos, equipamentos de saúde, materiais (órteses e próteses), hemoderivados, vacinas e insumos para diagnóstico de doenças, sendo que o déficit comercial acumulado passou de aproximadamente US\$ 700 milhões ao ano, no final da década de 1980, para um patamar superior a US\$ 9 bilhões em 2009. Destaque-se, também, que a vulnerabilidade da política nacional de saúde é influenciada por outros fatores tais como a pouca articulação entre os setores público e privado, a significativa defasagem tecnológica, as oscilações do mercado financeiro internacional e as estratégias competitivas globais das empresas que nem sempre são compatíveis com os interesses estratégicos nacionais.

Além disso, o crescimento da produção científica brasileira detentora da $15^{\text {a }}$ posição mundial na quantidade de publicações científicas em periódicos indexados em 2006 não foi acompanhada por igual desenvolvimento de inovações tecnológicas, como atesta o número de patentes produzidas no país (121 patentes, 28ª posição no ranking mundial em 2006), de modo que o grande desafio consiste em traduzir o conhecimento gerado em inovações que contribuam para melhorar o desempenho do setor produtivo nacional. 
Entretanto é importante salientar que algumas iniciativas destinadas a fortalecer a inovação tecnológica e o desenvolvimento produtivo dos setores que compõem o Ceis, buscando reduzir a dependência tecnológica externa, foram adotadas pelo governo brasileiro nos últimos anos, entre as quais cabe destacar a assunção do Ceis como área estratégica de desenvolvimento pela Pitce e pela Política de Desenvolvimento Produtivo, a criação da PNCT\&I/S, a elaboração da Agenda Nacional de Prioridades de Pesquisa em Saúde, o lançamento do Programa Mais Saúde (PAC Saúde), a definição por Portaria (MS n. 978, de 16/05/2008) da lista de produtos estratégicos no âmbito do SUS e a criação de linhas de financiamento voltadas para fortalecer os segmentos do Ceis por meio da Finep e do BNDES. No que diz respeito à fabricação de produtos médicos no Brasil, o estado de São Paulo ocupa lugar de destaque. Dados apresentados por Souza, Cappa e Neves (2008) mostram que $45,3 \%$ de todas as empresas brasileiras fabricantes de produtos médicos estão localizadas nesse estado, que responde por $56,6 \%$ de todos os postos de trabalho desse ramo industrial. A segmentação por grupos de produtos revela que o estado de São Paulo concentra 26,4\% das empresas de produtos farmoquímicos, 39,6\% das empresas que fabricam medicamentos para uso humano, $48,2 \%$ das empresas que produzem material médico, $46,9 \%$ das empresas fabricantes de aparelhos e instrumentos médicos e 66,4\% das empresas que produzem aparelhos e instrumentos de medida, teste e controle.

A concentração de Instituições de Ensino Superior (IES) no Brasil acompanha esse quadro. Do total de 2.281 IES brasileiras, 1.095 estão localizadas na região Sudeste, sendo que 547 estão sediadas no estado de São Paulo (Inep, 2007). Esse é um dos inúmeros exemplos da extrema desigualdade regional existente no território brasileiro, no que concerne a aspectos relacionados à ciência, tecnologia e inovação. 
A superação das desigualdades regionais é uma das estratégias da Política Nacional de Ciência, Tecnologia e Inovação em Saúde (Brasil, 2006), tendo como foco principal a articulação entre ações federais, estaduais e municipais no fomento de iniciativas de produção de conhecimento científico, fundamentadas nas necessidades regionais.

Além do seu impacto na capacidade de desenvolvimento humano e de dinamização da economia, Gadelha e Costa (2007) destacam que o Ceis também influencia, de forma significativa, o conteúdo da abordagem das questões referentes à integração regional e formação de blocos econômicos, na medida em que envolve um conjunto amplo de atividades que devem ser objeto de negociação entre os diferentes países, como o combate conjunto às epidemias e a circulação de produtos e serviços de saúde.

Além disso, o tema da integração regional tem estado cada vez mais presente na agenda econômica e política dos 70 países da América Latina, uma vez que esses países, num contexto de globalização assimétrica, buscam fortalecer seus vínculos para obter uma posição mais favorável nas negociações internacionais (Giovanella e Sanchez, 2007). O Mercosul representa uma importante iniciativa nesse sentido.

No caso da política de saúde, Sánches (2007) aponta a falta de definição de um modelo de integração regional em saúde no âmbito do Mercosul que seja capaz de orientar o processo de discussão em torno da saúde. Não se sabe, por exemplo, se o modelo a ser construído envolve a simples coordenação de serviços em zonas de fronteira ou a integração desses serviços; se o livre trânsito de pessoas (profissionais e usuários dos sistemas de saúde) constitui uma das metas da integração regional; ou como irá ocorrer o livre trânsito de produtos e serviços de saúde entre os países que integram o bloco regional.

É inegável o papel que as políticas de CT\&I vêm tendo no desenvolvimento dos países. Na área da saúde, extre- 
mamente dependente de medicamentos e equipamentos com altíssimos graus de CT\&I, essa questão aparece como central para as políticas públicas nacionais brasileiras e dos demais países da América do Sul, tendo em vista os déficits na balança comercial, já que grande parte dos procedimentos clínicos utiliza materiais, insumos e medicamentos importados. No caso do Brasil, para citar apenas um setor estratégico, o farmacêutico, além de o país apresentar um déficit comercial negativo crescente nos últimos cinco anos ${ }^{11}$, os dados de faturamento do setor em 2005, apontam que $60 \%$ de empresas que mais faturam são multinacionais. Esse fato aponta para um problema ainda mais grave uma vez que as empresas multinacionais realizam poucas atividades de $\mathrm{P} \& \mathrm{D}$ nas suas filiais, ficando todo o processo de inovação centralizado nos seus países de origem. Esforços estão sendo realizados nesse sentido pelo atual governo brasileiro para a diminuição dessa dependência e melhoria no sistema de desenvolvimento de CT\&I, sendo a saúde uma das principais áreas focalizadas por essas políticas.

No atual contexto mundial faz-se necessária a efetiva integração dos países da América do Sul, na medida em que o fortalecimento dessa região pode trazer grandes avanços no sentido de um desenvolvimento genuíno a fim de diminuir as históricas desigualdades de cada um dos países, assim como a extrema dependência dos países mais ricos.

$* * *$

O setor saúde constitui um lócus essencial de desenvolvimento econômico e social dadas suas possibilidades como

\footnotetext{
${ }^{11}$ Segundo dados da Febrafarama, o déficit comercial do setor farmacêutico no Brasil passou aproximadamente de US\$ 1,2 em 2003, para US\$ 2,7 em 2007 (Capanema, Palmeira Filho e Pieroni, 2008).
} 
espaço de inovação tecnológica, de acumulação de capital, de geração de emprego e renda bem como no campo da assistência pelas ações de promoção, manutenção e recuperação da saúde como qualificadoras da cidadania.

Para a renovação na formulação de estudos abrangendo a tríade saúde, desenvolvimento e inovação tecnológica, impõe-se como tarefa epistemológica central o resgate e a revisão do conceito de desenvolvimento à luz das experiências das políticas realizadas pelo Estado nas vertentes de bem-estar social e na de regulador analisadas sob a ótica das contribuições de teóricos estruturalistas cujo expoente brasileiro é Celso Furtado. Estudos assim concebidos certamente contribuirão de forma decisiva para a formulação da concepção de desenvolvimento aliada ao papel do Estado capaz de responder aos desafios contemporâneos postos ao setor saúde.

A análise a partir da articulação entre economia e polí72 tica social é essencial para a compreensão da relação entre política econômica e proteção social em sociedades com diversidades em contextos econômicos de modo a propiciar a identificação dos condicionantes e limites da integração entre as lógicas econômicas e de proteção social que sirvam de subsídio para o caso brasileiro.

Nessa perspectiva, vale mencionar a contribuição de Mkandwire acerca da nova economia do desenvolvimento que, ao contestar o modelo único de desenvolvimento do ideário liberal - o Estado como agente meramente regulador da atividade econômica -, tem o objetivo de libertar as forças criativas em busca de ampliar as opções à ação estatal. A ideia de desenvolvimento para países emergentes como o Brasil deve considerar as dimensões econômica e social, tendo por escopo o crescimento e modernização econômica, a melhoria na distribuição da riqueza e da renda, a garantia da soberania nacional, o fortalecimento da democracia e o estabelecimento do equilíbrio 
entre as regiões do país. Trata-se de renovar a concepção de desenvolvimento ressituando-o para além do crescimento econômico e da inovação tecnológica na direção do enfrentamento das desigualdades socioespaciais como base da cidadania.

No campo da investigação, os desenhos de estudos devem abarcar as políticas de ciência, tecnologia e inovação em saúde na perspectiva de descortinar a relação entre as políticas de saúde e os padrões de desenvolvimento, abordar a relação inovação/incorporação de tecnologias identificando experiências emblemáticas em busca de possibilidades na articulação entre pesquisa e produção e de fatores que condicionam o desempenho de instituições voltadas para a produção e pesquisa de tecnologias essenciais no Ceis. Para a consecução desse escopo, destaca-se a importância das investigações centradas no estado de São Paulo por sua expressividade no âmbito do complexo econômico industrial da saúde no país.

A nova perspectiva de abordagem e de investigação tem como fundamento último contribuir para o aperfeiçoamento da formulação e operacionalização de políticas públicas para induzir os setores do Ceis estratégicos para o Brasil coordenado ao aumento do poder de regulação do Estado, no estabelecimento de novos padrões de cooperação regional e ao fortalecimento de instituições públicas estratégicas na inovação de tecnologia na saúde.

\section{Ana Luiza d'Ávila Viana}

é professora do Departamento de Medicina Preventiva da Faculdade de Medicina da USP e pesquisadora do Cedec.

\section{Fabíola Lana Iozzi}

é doutoranda do Departamento de Medicina Preventiva da Faculdade de Medicina da USP. 


\section{Mariana Vercesi de Albuquerque}

é doutoranda do Departamento de Medicina Preventiva da Faculdade de Medicina da USP.

\section{Aylene Bousquat}

é professora do Mestrado em Saúde Coletiva da Universidade Católica de Santos e pesquisadora do Cedec.

\section{Referências bibliográficas}

\section{AGÊNCIA BRASILEIRA DE DESENVOLVIMENTO INDUSTRIAL}

[ABDI]. 2008a. Estudo prospectivo: equipamentos médicos, hospitalares e odontológicos. Brasília: Agência Brasileira de Desenvolvimento Industrial (Série Cadernos da Indústria ABDI, vol. VIII). . 2008b. Panorama setorial: equipamentos médicos, hospitalares e odontológicos. Brasília: Agência Brasileira de Desenvolvimento Industrial (Série Cadernos da Indústria ABDI, vol. VII).

ALMEIDA, E. P. 2005. Uso do território brasileiro e os serviços de saúde no período técnico-científico-informacional. Tese de Doutorado. São Paulo: FFLCH-USP.

BIELSCHOWSKY, R. 2000a. Pensamento econômico brasileiro: o ciclo ideológico do desenvolvimentismo. Rio de Janeiro: Contraponto. (org.). 2000b. Cinquenta anos de pensamento na Cepal. Rio de Janeiro: Campus.

BRASIL. 2006. Política nacional de ciência, tecnologia e inovação em saúde. 2.ed. Brasília: Ministério da Saúde (Série B: Textos Básicos em Saúde). BRESSER-PEREIRA, L. C. 2007. "Novo desenvolvimentismo e ortodoxia convencional" (mimeo.).

CAPANEMA, L. X. L.; PALMEIRA FILHO, P. L; PIERONI, J. P. 2008. "Apoio do BNDES a Complexo Industrial da Saúde: experiência do Profarma e seus desdobramentos”. BNDES Setorial, n. 27, pp. 3-20.

CEALAG. 2007. "Relatório preliminar da pesquisa Estudo dos Departamentos Regionais de Saúde da Secretaria de Estado de Saúde de São Paulo" (mimeo).

COHN, A. 2005. "O SUS e o direito à saúde: universalização e focalização nas políticas de saúde”. In: LIMA, N. T. (org.). Saúde e democracia: história e perspectivas do SUS. Rio de Janeiro: Ed. Fiocruz.

CONASS. 2007. Ciência e tecnologia em saúde. Brasília: Conass (Coleção Progestores: Para Entender a Gestão do SUS, vol. 4).

CORDEIRO, H. 1985. A indústria da saúde no Brasil. 2.ed. Rio de Janeiro: Graal. 
COUTINHO, L. 2000. "O desafio urbano-regional na construção de um projeto de nação”. In: GONÇALVES, M. F.; BRANDÃO, C. A.; GALVÃO, A. C. F. (orgs.). Regiões e cidades, cidades nas regiões: o desafio urbano-regional. São Paulo: Ed. Unesp/Anpur.

DRAIBE, S. M. 1997. "Uma nova institucionalidade das políticas sociais? Reflexões a respeito da experiência latino-americana recente de reformas dos programas sociais". São Paulo em Perspectiva, vol. 11, n. 4, pp. 3-15. . 2007a. "Coesão social e integração regional". Cadernos de Saúde Pública, vol. 23, sup. 2, pp. 174-183. . 2007b. "Estado de bem-estar, desenvolvimento econômico e cidadania: algumas lições da literatura contemporânea”. In: HOCHMAN, G. (org.). Políticas públicas no Brasil. Rio de Janeiro: Ed. Fiocruz.

FIPE; IPT. 2008. Uma agenda de competitividade para a indústria paulista de equipamentos médico-hospitalares e odontológicos. São Paulo: Fipe/IPT.

FURTADO, C. 1974. O mito do desenvolvimento econômico. Rio de Janeiro: Paz e Terra.

FURTADO, J. 2001. "A indústria de equipamentos médico-hospitalares: elementos para uma caracterização da sua dimensão internacional”. In: NEGRI, B.; DI GIOVANNI, G. (orgs.). Brasil: radiografia da saúde. Campinas: Ed. Unicamp.

GADELHA, C. A. G. 2001. "Política industrial: uma visão neosschumpeteriana sistêmica e estrutural”. Revista de Economia Política, vol. 21, n. 4, pp. 149-171. . 2002. "Estado e inovação: uma perspectiva evolucionista". Revista de Economia Contemporânea, vol. 6, n. 2, pp. 85-117. . 2003. "O complexo industrial da saúde e a necessidade de um enfoque dinâmico na economia da saúde". Ciência Ẽ Saúde Coletiva, vol. 8, n. 2, pp. 521-535. . 2006. "Desenvolvimento, complexo industrial da saúde e política industrial”. Revista de Saúde Pública, vol. 40, n. esp., pp. 11-23. ; ROMERO, C. 2007. "Complexo industrial da saúde e inovação: desafios para a competitividade nacional em vacinas e o papel da Fiocruz”. In: AZEVEDO, N. et al. (orgs.). Inovação em saúde: dilemas e desafios de uma instituição pública. Rio de Janeiro: Ed. Fiocruz. ; COSTA, L. 2007. "Integração de fronteiras: a saúde no contexto de uma política nacional de desenvolvimento". Cadernos de Saúde Pública, vol. 23, sup. 2, pp. 214-226.

GARCIA, M. A. 2008. "A opção sul-americana”. Revista Interesse Nacional, ano I, n. 1, pp. 45-52. 
GELIJNS, A. C.; ROSEMBERG, N. 1995. "The changing nature of medical technology development”. In: ROSEMBERG, N.; GELIJNS A. C.; DAWKINS, H. (orgs.). Sources of medical technology: universities and industry. Washington: National Academy Press.

GIOVANELLA, L.; SÁNCHES, D. 2007. "Editorial: integración regional y políticas de salud”. Cadernos de Saúde Pública, vol. 23, sup. 2, pp. 114-115.

LEHOUX, P. 2002. Une analyse critique de la valeur des technologies et des processus innovants: peut-elle nous amener à concevoir de nouveaux instruments de réguation? Montréal: Université de Montréal. et al. 2008. "What leads to better health care innovation? Arguments for an integrated policy-oriented research agenda”. Journal of Health Services Research Eं Policy, vol. 13, n. 4, pp. 251-254.

MACHADO, C. V. 2007. Direito universal, política nacional: o papel do Ministério da Saúde na política de saúde brasileira de 1990 a 2002. Rio de Janeiro: Ed. Museu da República.

NEGRI, B.; GIOVANNI, G. (orgs.) 2001. Brasil: radiografia da saúde. Campinas: Ed. Unicamp.

MATTOS, P. T. L. 2006. "A formação do Estado regulador". Novos Estudos Cebrap, n. 76, p. 139-156.

NOVAES, H. M. D. 2006. "Da produção à avaliação de tecnologias dos sistemas de saúde: desafios do século XXI”. Revista de Saúde Pública, vol. 40, n. esp., pp. 133-140.

SÁNCHES, D. M. 2007. "Procesos de integración en salud: sobre los desafios del Mercosur en salud". Cadernos de Saúde Pública, vol. 23, sup. 2, pp. 155-163.

SANTOS, W. G. 1979. Cidadania e justiça. Rio de Janeiro: Campus.

SEN, A. 2000. Desenvolvimento como liberdade. São Paulo: Companhia das Letras. SISCÚ, J.; DE PAULA, L. F.; RENAULT, M. 2007. "Por que novo-desenvolvimentismo?”. Revista de Economia Política, vol. 27, n. 4, pp. 507-524.

SOUZA, J. H.; CAPPA, J.; NEVES, L. C. 2008. "Concentração regional da indústria de produtos médicos”. São Paulo em Perspectiva, vol. 22, n. 1, pp. 123-136.

VIANA, A. L. D’A. et al. 2007a. "Economia política da saúde: introduzindo o debate”. Saúde para Debate, vol. 37, pp. 16-39. et al. 2007b."Sistema de saúde universal e território: desafios de uma política regional para a Amazônia Legal”. Cadernos de Saúde Pública, vol. 23, sup. 2, p. 117-131. ; ELIAS, P. E. 2007. "Saúde e desenvolvimento". Ciência Ẽ Saúde Coletiva, vol. 12, p. 1765-1777. 


\section{Outros materiais}

BNDES. 2008. Seminário Complexo Econômico-Industrial da Saúde: em Busca de uma Articulação entre Inovação, Acesso e Desenvolvimento Industrial. 19-21 mai. 2008. Disponível em: < http:/ /www.bndes.gov.br/SiteBNDES/ bndes/bndes_pt/Institucional/Publicacoes/Paginas/s_saude3.html>. Acesso em: 03/07/2011.

BRASIL. 2007. Discurso pronunciado pelo Ministro de Estado da Saúde, José Gomes Temporão. XIII Conferência Nacional de Saúde. Brasília, 14-18 nov. 2007. Disponível em: <http://www.ensp.fiocruz.br/biblioteca/ home/exibedetalhesBiblioteca.cfm?ID $=4774 \&$ Tipo $=\mathrm{B}>$. Acesso em: $15 / 08 / 2010$.

INEP. 2007. Censo Educação Superior. Disponível em: <www.inep.gov.br/ superior/censosuperior/sinopse>. Acesso em: 01/07/2009.

MINISTÉRIO DA SAÚDE. 2008. "Saúde e desenvolvimento: diretrizes estratégicas". Disponível em: <http://bvsms.saude.gov.br/bvs/ publicacoes/mais_saude_diretrizes.pdf >. Acesso em: 03/07/2011. MKANDAWIRE, T. 2002. "Le retour du développement". Courrier de la Planète: Développment Environnement, les Défis d'ún Monde Solidaire, $\mathrm{n}$. 67, vol. 1. Disponível em: <http://www.unrisd.org/unrisd/website/ newsview.nsf/0/154BDB05902D3BD9C1256>. Acesso em: 10/03/2011. 


\section{SAÚDE, DESENVOLVIMENTO E INOVAÇÃO TECNOLÓGICA: NOVA PERSPECTIVA DE ABORDAGEM E DE INVESTIGAÇÃO}

ANA LUIZA D’ÁVILA VIANA

FABÍOLA LANA IOZZI

MARIANA VERCESI DE ALBUQUERQUE

\section{AYLENE BOUSQUAT}

O presente artigo tem como objetivo contribuir para a renovação na formulação de estudos que abrangem a tríade saúde, desenvolvimento e inovação tecnológica. A tarefa epistemológica central é o resgate e revisão do conceito de desenvolvimento à luz das experiências das políticas desenvolvidas pelo Estado na vertente de bem-estar social e na de regulador analisadas sob a ótica das contribuições de teóricos estruturalistas. A nova perspectiva de investigação tem como fundamento último contribuir para o aperfeiçoamento da formulação e operacionalização de políticas públicas para induzir os setores do complexo econômico-industrial da saúde estratégicos para o Brasil, coordenado ao aumento do poder de regulação do Estado e ao fortalecimento de instituições públicas estratégicas na inovação de tecnologia na saúde.

Palavras-chave: Saúde, política; desenvolvimento; inovação tecnológica, política

\section{HEALTHCARE, DEVELOPMENT AND TECHNOLOGY INNOVATION: A NEW INVESTIGATION FIELD}

This article aims to contribute to the renewal in the formulation of studies covering the triad health, development and technological innovation. The central epistemological task is the rescue and review of the development concept in light of experiences of policies 
pursued by theorical framework of social welfare and the regulator analyzed from the perspective of the contributions of structuralist theorists. The new perspective and approach to research is based on the latter contribute to improving the design and operation of public policies to induce economic sectors of the health-industrial complex strategic for Brazil, coordinated the increased power of government regulation and strengthening public institutions in strategic technology innovation in healthcare.

Keywords: Health policies; development; technology innovation; policies

\section{RELAÇÕES ENTRE EQUIDADE E VIABILIDADE NOS EMPREENDIMENTOS SOLIDÁRIOS}

\section{LUIZ INÁCIO GAIGER}

A economia solidária pode ser considerada como uma alternativa para a geração de renda, para o combate à pobreza e para a ruptura com a lógica social da desigualdade, desde que promova o protagonismo dos pobres, o qual é necessário para que intervenções políticas nesse campo sejam eficazes. $\mathrm{O}$ artigo aborda esse tema mediante o exame de evidências colhidas em pesquisas empíricas e por meio de uma exploração dos dados finais do Primeiro Mapeamento Nacional da Economia Solidária no Brasil. Ao comparar os empreendimentos solidários com as características gerais das empresas no país, segundo as estatísticas nacionais, sobressaem-se singularidades dos empreendimentos solidários, como a propensão a preservar os postos de trabalho e a manter princípios igualitários na distribuição dos rendimentos e benefícios decorrentes de suas atividades econômicas. Tais regimes de equidade, presentes em sua gênese, são constitutivos de sua forma social de produção. 